\title{
A unified formula for Steenrod operations in flag manifolds
}

\author{
Haibao Duan and Xuezhi Zhao
}

\begin{abstract}
The classical Schubert cells on a flag manifold $G / P$ give a cell decomposition for $G / P$ whose Kronecker duals (known as Schubert classes) form an additive base for the integral cohomology $H^{*}(G / P)$. We present a formula that expresses Steenrod mod- $p$ operations on Schubert classes in $G / P$ in terms of Cartan numbers of $G$.
\end{abstract}

\section{Introduction}

For a prime $p \geqslant 2$, let $\mathcal{A}_{p}$ be the mod- $p$ Steenrod algebra, and let $\mathcal{P}^{k} \in \mathcal{A}_{p}, k \geqslant 1$, be the Steenrod mod- $p$ reduced powers on the $\mathbb{F}_{p}$-cohomology of topological spaces [SE62] (here $\mathbb{F}_{p}=\mathbb{Z} / p \mathbb{Z}$ ). In the case $p=2$, it is also customary to use $S q^{2 k} \in \mathcal{A}_{2}$ instead of $\mathcal{P}^{k}$.

An entire description of the $\mathcal{A}_{p}$-action on the $\mathbb{F}_{p}$-cohomology of a topological space $X$ leads to two enquiries.

Problem A. Specify an additive basis $S=\left\{\omega_{1}, \ldots, \omega_{m}\right\}$ for the graded $\mathbb{F}_{p}$-vector space $H^{*}\left(X ; \mathbb{F}_{p}\right)$ that encodes the geometric formation of $X$ (e.g. a cell decomposition of $X$ ).

Problem B. Determine the coefficients $c_{i, j}^{k} \in \mathbb{F}_{p}$ in the expression

$$
\mathcal{P}^{k}\left(\omega_{i}\right)=\sum_{1 \leqslant j \leqslant m} c_{i, j}^{k} \omega_{j}, \quad k \geqslant 1,1 \leqslant i \leqslant m .
$$

The study of the internal structure of the algebra $\mathcal{A}_{p}$ has continued for almost 50 years, see Wood [Woo98] for a thorough historical account, further problems and relevant references. On the other hand, we note that even partial solutions to Problem B can have significant consequences in manifold geometry: the classical Wu formula [Wu50] can be interpreted as the expansion of the $S q^{k}$-action on the special Schubert classes in the real Grassmannians; the calculation by Steenrod and Whitehead [SW51] in the truncated real projective spaces led to an enormous step in understanding the problem of how many linearly independent vector fields can be found on the $n$-sphere $S^{n}$; by deriving partial knowledge of the $\mathcal{P}^{k}$-action on the special Schubert classes in the complex Grassmannian, Borel and Serre [BS51, BS53] demonstrated that the $2 n$-dimensional spheres $S^{2 n}$ do not admit any almost complex structure unless $n=1,2,3$. Needless to say, there are many profound applications and deep implications of Steenrod operations in topology [Die89, Len98, Ste72], and effective calculation of these operations on the cohomology of a given manifold also deserves high priority.

Let $G$ be a compact connected Lie group and let $P$ be the centralizer of a one-parameter subgroup in $G$. The space $G / P=\{g P \mid g \in G\}$ of left cosets of $P$ in $G$ is known as a flag manifold. In this paper, we study Problems A and B for all $G / P$.

Received 14 February 2005, accepted in final form 10 May 2006.

2000 Mathematics Subject Classification 55S10, 14M10, $14 \mathrm{M} 15$.

Keywords: Steenrod operations, flag manifolds, Schubert cells, Cartan numbers.

The first author is supported by NSFC.

This journal is (C) Foundation Compositio Mathematica 2007. 


\section{H. DUAN AND X. ZHAO}

Firstly, if $X$ is a flag manifold $G / P$, a uniform solution to Problem A is already known as the basis theorem in Schubert's enumerative calculus [Kle76, Sot02, BGG73]. This was originated by Ehresmann [Ehr34] for the Grassmannians $G_{n, k}$ of $k$-dimensional subspaces in $\mathbb{C}^{n}$ in 1934 , extended to the case where $G$ is a matrix group by Bruhat in 1954, and completed for all compact connected Lie groups by Chevalley [Che94] in 1958. We briefly recall the result.

Let $W$ and $W^{\prime}$ be the Weyl groups of $G$ and $P$, respectively. The set $W / W^{\prime}$ of left cosets of $W^{\prime}$ in $W$ can be identified with the subset of $W$ :

$$
\bar{W}=\left\{w \in W \mid \ell\left(w_{1}\right) \geqslant \ell(w) \text { for all } w_{1} \in w W^{\prime}\right\}
$$

where $\ell: W \rightarrow \mathbb{Z}$ is the length function relative to a fixed maximal torus $T$ in $G$ [BGG73, Proposition 5.1]. The key fact is that the space $G / P$ admits a canonical decomposition into cells indexed by elements of $\bar{W}$

$$
G / P=\bigcup_{w \in \bar{W}} X_{w}, \quad \operatorname{dim} X_{w}=2 \ell(w),
$$

where each cell $X_{w}$ is the closure of an algebraic affine space, known as a Schubert variety on $G / P$ (see [Che94, BGG73]). Since only even-dimensional cells are involved in the decomposition (1.1), the set of fundamental classes $\left[X_{w}\right] \in H_{2 \ell(w)}(G / P), w \in \bar{W}$, forms an additive basis of the homology $H_{*}(G / P)$. The cocycle class $\sigma_{w} \in H^{2 \ell(w)}(G / P)$ defined by the Kronecker pairing as

$$
\left\langle\sigma_{w},\left[X_{u}\right]\right\rangle=\delta_{w, u}, \quad w, u \in \bar{W}
$$

is called the Schubert class corresponding to $w$. Combining (1.1) with the Poincaré duality yields a solution to Problem A.

Lemma 1 (Basis theorem). The set of Schubert classes $\left\{\sigma_{w} \mid w \in \bar{W}\right\}$ constitutes an additive basis for the ring $H^{*}(G / P)$.

It follows from Lemma 1 that, for a $u \in \bar{W}$ and $k \geqslant 1$, one has the expression

$$
\mathcal{P}^{k}\left(\sigma_{u}\right) \equiv \sum a_{u, w}^{k} \sigma_{w} \quad \bmod p, \quad a_{u, w}^{k} \in \mathbb{F}_{p},
$$

where the sum ranges over all $w \in \bar{W}$ with $\ell(w)=\ell(u)+k(p-1)$ since $\mathcal{P}^{k}$ has degree $k(p-1)$. Thus, in the case of $X=G / P$, Problem B admits a concrete form.

Problem B'. Determine the numbers $a_{u, w}^{k} \in \mathbb{F}_{p}$ for $k \geqslant 1, w, u \in \bar{W}$ with $\ell(w)=\ell(u)+k(p-1)$.

If $G$ is the unitary group $U(n)$ of order $n$ and if $P=U(k) \times U(n-k)$, the flag manifold $G / P$ is the complex Grassmannian $G_{n, k}$ of $k$-planes through the origin in $\mathbb{C}^{n}$. The $i$ th Chern classes $c_{i} \in H^{2 i}\left(G_{n, k}\right), 1 \leqslant i \leqslant k$, of the canonical complex $k$-bundle over $G_{n, k}$ are precisely the special Schubert classes on $G_{n, k}$ (see [Sot02, GH78]). In order to generalize the Wu formula [Wu50], many works were devoted to finding an expression of $\mathcal{P}^{k}\left(c_{i}\right)$ in terms of the $c_{i}$ (cf. [BS51, BS53, Dua03b, Lan83, Len98, Pet75, Sha77, Sug79]). This seems to be the only special case for which Problem B' has been studied in some detail.

It is well known that the knowledge of the $\mathcal{A}_{p}$ action on the $\mathbb{F}_{p}$ cohomology of a space $X$ can provide deeper information on the topology of $X$ than just the cohomology ring structure. In particular, in view of the geometric decomposition (1.1) of $G / P$ offered by the classical Schubert cells, the numbers $a_{w, u}^{k}$ are immediately applicable to investigating the attaching maps of these cells (e.g. compare the tables in $\S 5$ with the figure in [Len98, $\S 6]$ ). We quote from Lenart [Len98] for the case of $G_{n, k}$ : 'Apart from projective spaces, very little is known about the attaching maps of their cells'.

The present paper is a sequel to [Dua05, DZ03], where the multiplicative rule of Schubert classes was determined. There is a common main idea behind these works. The calculation in 


\section{Formula For SteEnRod Operations}

the ring $H^{*}(G / P)$ can be brought to the cohomology of an appropriate Bott-Samelson $K$-cycle (cf. Definition 4), which provides the desingularization of a Schubert variety.

This paper is so arranged as follows. Section 2 contains a brief introduction to the Weyl group associated with a Lie group. Then the solution to Problem $\mathrm{B}^{\prime}$ is presented in the Theorem. After geometric preliminaries in $\S 3$, the Theorem is established in $\S 4$. In order to illustrate the effective computability of our method, computational results for some cases of $G / P$ are explained and tabulated in $\S 5$.

\section{The result}

To investigate the topology of a flag manifold $G / P$ one may assume that the Lie group $G$ under consideration is 1-connected and semi-simple [BH58]. Since all 1-connected semi-simple Lie groups are classified by their Cartan matrices [Hum72, p. 55], any numerical topological invariant of $G / P$ may be reduced to the Cartan numbers (entries in the Cartan matrix of $G$ ). We present both a formula and an algorithm that evaluate the numbers $a_{u, w}^{k} \in \mathbb{F}_{p}$ in terms of Cartan numbers of $G$.

Fix a maximal torus $T$ of $G$ and set $n=\operatorname{dim} T$. Equip the Lie algebra $L(G)$ of $G$ with an inner product $(\cdot, \cdot)$ so that the adjoint representation acts as isometries of $L(G)$. The Cartan subalgebra of $G$ is the Euclidean subspace $L(T)$ of $L(G)$.

The restriction of the exponential map exp: $L(G) \rightarrow G$ to $L(T)$ defines a set $D(G)$ of $\frac{1}{2}(\operatorname{dim} G-n)$ hyperplanes in $L(T)$, i.e. the set of singular hyperplanes through the origin in $L(T)$. The reflections of $L(T)$ in these planes generate the Weyl group $W$ of $G$ (see [Hum72, p. 49]).

Fix a regular point $\alpha \in L(T) \backslash \bigcup_{L \in D(G)} L$ and let $\Delta$ be the set of simple roots relative to $\alpha$ [Hum72, p. 47]. If $\beta \in \Delta$, the reflection $s_{\beta}$ in the hyperplane $L_{\beta} \in D(G)$ relative to $\beta$ is called a simple reflection. If $\beta, \beta^{\prime} \in \Delta$, the Cartan number

$$
\beta \circ \beta^{\prime}=2\left(\beta, \beta^{\prime}\right) /\left(\beta^{\prime}, \beta^{\prime}\right)
$$

is always an integer (only $0, \pm 1, \pm 2, \pm 3$ can occur [Hum72, p. 55]).

Since the set of simple reflections $\left\{s_{\beta} \mid \beta \in \Delta\right\}$ generates $W$, any $w \in W$ admits a factorization of the form

$$
w=s_{\beta_{1}} \circ \cdots \circ s_{\beta_{m}}, \quad \beta_{i} \in \Delta .
$$

Definition 1. The length $\ell(w)$ of a $w \in W$ is the lowest number of factors in all decompositions of $w$ in the form (2.1). The decomposition (2.1) is said reduced if $m=\ell(w)$.

If (2.1) is a reduced decomposition, the $m \times m$ (strictly upper triangular) matrix $A_{w}=\left(a_{i, j}\right)$ with

$$
a_{i, j}= \begin{cases}0 & \text { if } i \geqslant j \\ -\beta_{j} \circ \beta_{i} & \text { if } i<j\end{cases}
$$

will be called the Cartan matrix of $w$ associated to the decomposition (2.1).

Let $\mathbb{Z}\left[x_{1}, \ldots, x_{m}\right]=\bigotimes_{n \geqslant 0} \mathbb{Z}\left[x_{1}, \ldots, x_{m}\right]^{(n)}$ be the ring of integral polynomials in $x_{1}, \ldots, x_{m}$, graded by $\left|x_{i}\right|=1$.

Definition 2. For a subsequence $\left[i_{1}, \ldots, i_{r}\right] \subseteq[1, \ldots, m]$ and $1 \leqslant k \leqslant r$, denote by $m_{k, p}\left(x_{i_{1}}, \ldots, x_{i_{r}}\right)$ the polynomial

$$
\sum_{\left(\alpha_{1}, \ldots, \alpha_{r}\right)} x_{i_{1}}^{\alpha_{1}} \cdots x_{i_{r}}^{\alpha_{r}} \in \mathbb{Z}\left[x_{1}, \ldots, x_{m}\right]^{(r+k(p-1))},
$$

where the sum is over all distinct permutations $\left(\alpha_{1}, \ldots, \alpha_{r}\right)$ of the partition $\left(p^{k}, 1^{r-k}\right)$ (see [Mac95, p. 1]). 


\section{H. DUAN AND X. ZhaO}

Remark 1 . In the theory of symmetric functions, $m_{k, p}\left(x_{i_{1}}, \ldots, x_{i_{r}}\right)$ is known as the monomial symmetric function in $x_{i_{1}}, \ldots, x_{i_{r}}$ associated to the partition $\left(p^{k}, 1^{r-k}\right)$ (see [Mac95, p. 19]). As examples, if $\left[i_{1}, \ldots, i_{r}\right]=[1,2,3]$, one has

$$
\begin{gathered}
m_{1, p}\left(x_{i_{1}}, \ldots, x_{i_{r}}\right)=x_{1}^{p} x_{2} x_{3}+x_{1} x_{2}^{p} x_{3}+x_{1} x_{2} x_{3}^{p}, \\
m_{2, p}\left(x_{i_{1}}, \ldots, x_{i_{r}}\right)=x_{1}^{p} x_{2}^{p} x_{3}+x_{1} x_{2}^{p} x_{3}^{p}+x_{1}^{p} x_{2} x_{3}^{p}, \\
m_{3, p}\left(x_{i_{1}}, \ldots, x_{i_{r}}\right)=x_{1}^{p} x_{2}^{p} x_{3}^{p} .
\end{gathered}
$$

Definition 3. Given an $m \times m$ strictly upper triangular integer matrix $A=\left(a_{i, j}\right)$ define an additive homomorphism $T_{A}: \mathbb{Z}\left[x_{1}, \ldots, x_{m}\right]^{(m)} \rightarrow \mathbb{Z}$ recursively as follows:

(1) for $h \in \mathbb{Z}\left[x_{1}, \ldots, x_{m-1}\right]^{(m)}, T_{A}(h)=0$;

(2) if $m=1$ (consequently, $A=(0)$ ), then $T_{A}\left(x_{1}\right)=1$;

(3) for $h \in \mathbb{Z}\left[x_{1}, \ldots, x_{m-1}\right]^{(m-r)}$ with $r \geqslant 1$,

$$
T_{A}\left(h x_{m}^{r}\right)=T_{A^{\prime}}\left(h\left(a_{1, m} x_{1}+\cdots+a_{m-1, m} x_{m-1}\right)^{r-1}\right),
$$

where $A^{\prime}$ is the $((m-1) \times(m-1)$ strictly upper triangular $)$ matrix obtained from $A$ by deleting the $m$ th column and the $m$ th row.

By additivity, $T_{A}$ is defined for every $h \in \mathbb{Z}\left[x_{1}, \ldots, x_{m}\right]^{(m)}$ using the unique expansion $h=$ $\sum_{0 \leqslant r \leqslant m} h_{r} x_{m}^{r}$ with $h_{r} \in \mathbb{Z}\left[x_{1}, \ldots, x_{m-1}\right]^{(m-r)}$.

Remark 2. Definition 3 implies an effective algorithm to evaluate $T_{A}$.

For $k=2$ and $A_{1}=\left(\begin{array}{ll}0 & a \\ 0 & 0\end{array}\right), T_{A_{1}}: \mathbb{Z}\left[x_{1}, x_{2}\right]^{(2)} \rightarrow \mathbb{Z}$ is given by

$$
\begin{gathered}
T_{A_{1}}\left(x_{1}^{2}\right)=0, \quad T_{A_{1}}\left(x_{1} x_{2}\right)=T_{A_{1}}\left(x_{1}\right)=1 \\
T_{A_{1}}\left(x_{2}^{2}\right)=T_{A_{1}}\left(a x_{1}\right)=a .
\end{gathered}
$$

For $k=3$ and $A_{2}=\left(\begin{array}{lll}0 & a & b \\ 0 & 0 & c \\ 0 & 0 & 0\end{array}\right), A_{2}^{\prime}=A_{1}$ and $T_{A_{2}}: \mathbb{Z}\left[x_{1}, x_{2}, x_{3}\right]^{(3)} \rightarrow \mathbb{Z}$ is given by

$$
T_{A_{2}}\left(x_{1}^{r_{1}} x_{2}^{r_{2}} x_{3}^{r_{3}}\right)= \begin{cases}0, & \text { if } r_{3}=0, \\ T_{A_{1}}\left(x_{1}^{r_{1}} x_{2}^{r_{2}}\left(b x_{1}+c x_{2}\right)^{r_{3}-1}\right), & \text { if } r_{3} \geqslant 1,\end{cases}
$$

where $r_{1}+r_{2}+r_{3}=3$, and where $T_{A_{1}}$ is calculated in the above.

Assume that $w=s_{\beta_{1}} \circ \cdots \circ s_{\beta_{m}}, \beta_{i} \in \Delta$, is a reduced decomposition of $w \in \bar{W}$ and let $A_{w}=\left(a_{i, j}\right)_{m \times m}$ be the associated Cartan matrix of $w$. For a subsequence $J=\left[i_{1}, \ldots, i_{r}\right] \subseteq[1, \ldots, m]$ we set

$$
s_{J}=s_{\beta_{i_{1}}} \circ \cdots \circ s_{\beta_{i_{r}}} \in W .
$$

A solution to Problem $\mathrm{B}^{\prime}$ is the following.

Theorem. For a $u \in \bar{W}, k>1$ with $\ell(u)=r$ and $m=r+k(p-1)$, we have (in (1.2)) that

$$
a_{u, w}^{k} \equiv T_{A_{w}}\left(\sum_{J=\left[i_{1}, \ldots, i_{r}\right] \subseteq[1, \ldots, m]} m_{k, p}\left(x_{i_{1}}, \ldots, x_{i_{r}}\right)\right) \bmod p .
$$

This result indicates an effective algorithm to evaluate $a_{u, w}^{k}$ as the following recipe shows:

(1) starting from the Cartan matrix of $G$, a program to present all elements of $\bar{W}$ by their minimal reduced decompositions is available in [DZ03, DZZ04]; with this presentation, $\bar{W}$ also becomes an ordered set (cf. $\S 5$ );

(2) for a $w \in \bar{W}$ with a reduced decomposition, the corresponding Cartan matrix $A_{w}$ can be read directly from Cartan matrix of $G$ (compare Definition 1 with [Hum72, p. 59]); 


\section{Formula FOR SteEnRod OpERATions}

(3) for a $w \in \bar{W}$ with a reduced decomposition $w=s_{\beta_{1}} \circ \cdots \circ s_{\beta_{m}}$ and a $u \in \bar{W}$ with $\ell(u)=r<m$, the solutions in the subsequence $J=\left[i_{1}, \ldots, i_{r}\right] \subseteq[1, \ldots, m]$ to the equation $s_{J}=u$ in $W$ agree with the solutions to the equation $s_{J}(\alpha)=u(\alpha)$ in the vector space $L(T)$, where $\alpha \in L(T)$ is a fixed regular point;

(4) the evaluation the operator $T_{A_{w}}$ on a polynomial can be easily programmed (cf. Definition 3 or $[\mathrm{DZ} 03, \S 5])$.

Based on the algorithm explained above, a parallel program to give the full expansion

$$
\mathcal{P}^{k}\left(\sigma_{u}\right) \equiv \sum a_{u, w}^{k} \sigma_{w} \bmod p
$$

has been implemented (in which the order on $\bar{W}$ is used in assigning to each $w \in \bar{W}$ a computing unit). We refer to $\S 5$ for some computational results from the program.

\section{Preliminaries in the $K$-cycles of Bott-Samelson}

In this section all homologies (respectively cohomologies) will have integer coefficients unless otherwise specified. If $f: X \rightarrow Y$ is a continuous map between two topological spaces, $f_{*}$ (respectively $f^{*}$ ) is the homology (respectively cohomology) homomorphism induced by $f$. If $M$ is an oriented closed manifold (respectively a connected projective variety) $[M] \in H_{\operatorname{dim} M}(M)$ stands for the orientation class. The Kronecker pairing, between cohomology and homology of a space $X$, will be denoted by $\langle\cdot, \cdot\rangle: H^{*}(X) \times H_{*}(X) \rightarrow \mathbb{Z}$.

The proof of the Theorem will make use of the celebrated $K$-cycles (i.e. Bott-Samelson resolutions of Schubert varieties) on the flag manifold $G / T$ constructed by Bott and Samelson early in 1955 [BS55]. In this section we recall the construction of these cycles, as well as their basic properties (from Lemmas 2-4) developed in [BS55, BS58, Dua03a, Dua05]. The main technical result in this section is Lemma 5, which allows us to transform the proof of the Theorem for $G / P$ to a calculation in terms of the $K$-cycles of Bott-Samelson.

As in $\S 2$, we fix a regular point $\alpha \in L(T)$ and let $\Delta$ be the set of simple roots relative to $\alpha$. For a $\beta \in \Delta$, the singular plane in $L(T)$ relative to $\beta$ will be denoted by $L_{\beta}$ (see [Hum72, p. 47]). Denote by $K_{\beta}$ the centralizer of $\exp \left(L_{\beta}\right)$ in $G$, where exp is the restriction of the exponential map $L(G) \rightarrow G$ to $L(T)$. We note that $T \subset K_{\beta}$ and that the quotient manifold $K_{\beta} / T$ is diffeomorphic to a 2 -sphere [BS58, p. 996].

The 2-sphere $K_{\beta} / T$ carries a natural orientation $\omega_{\beta} \in H^{2}\left(K_{\beta} / T ; \mathbb{Z}\right)$ that may be specified as follows. The Cartan decomposition of the Lie algebra $L\left(K_{\beta}\right)$ relative to the maximal torus $T \subset K_{\beta}$ has the form $L\left(K_{\beta}\right)=L(T) \oplus \vartheta_{\beta}$, where $\vartheta_{\beta} \subset L(G)$ is a 2-plane, the root space belonging to the root $\beta$ (see $\left[\right.$ Hum72, p. 35]). Let $[\cdot, \cdot]$ be the Lie bracket on $L(G)$. Take a non-zero vector $v \in \vartheta_{\beta}$ and let $v^{\prime} \in \vartheta_{\beta}$ be such that $\left[v, v^{\prime}\right]=\beta$. The ordered base $\left\{v, v^{\prime}\right\}$ gives an orientation on $\vartheta_{\beta}$ which does not depend on the initial choice of $v$.

The tangential of the quotient map $\pi_{\beta}: K_{\beta} \rightarrow K_{\beta} / T$ at the group unit $e \in K_{\beta}$ maps $\vartheta_{\beta}$ isomorphically onto the tangent space to $K_{\beta} / T$ at $\pi_{\beta}(e)$. In this manner the orientation $\left\{v, v^{\prime}\right\}$ on $\vartheta_{\beta}$ furnishes $K_{\beta} / T$ with the induced orientation $\omega_{\beta}=\left\{\pi_{\beta}(v), \pi_{\beta}\left(v^{\prime}\right)\right\}$.

For a sequence $\beta_{1}, \ldots, \beta_{m} \in \Delta$ of simple roots (repetitions $\beta_{i}=\beta_{j}$ may occur), let $K\left(\beta_{1}, \ldots, \beta_{m}\right.$ ) be the product group $K_{\beta_{1}} \times \cdots \times K_{\beta_{m}}$. Since $T \subset K_{\beta_{i}}$ for each $i$ the group $T \times \cdots \times T$ (m-copies) acts on $K\left(\beta_{1}, \ldots, \beta_{m}\right)$ from the right by

$$
\left(g_{1}, \ldots, g_{m}\right)\left(t_{1}, \ldots, t_{m}\right)=\left(g_{1} t_{1}, t_{1}^{-1} g_{2} t_{2}, \ldots, t_{m-1}^{-1} g_{m} t_{m}\right) .
$$

Let $\Gamma\left(\beta_{1}, \ldots, \beta_{m}\right)$ be the base manifold of this principal action, oriented by the $\omega_{\beta_{i}}, 1 \leqslant i \leqslant m$. The point in $\Gamma\left(\beta_{1}, \ldots, \beta_{m}\right)$ corresponding to a $\left(g_{1}, \ldots, g_{m}\right) \in K\left(\beta_{1}, \ldots, \beta_{m}\right)$ denoted by $\left[g_{1}, \ldots, g_{m}\right]$. 
The integral cohomology of $\Gamma\left(\beta_{1}, \ldots, \beta_{m}\right)$ has been determined in [BS55, Proposition II]. Let $\varphi_{i}: K_{\beta_{i}} / T \rightarrow \Gamma\left(\beta_{1}, \ldots, \beta_{m}\right)$ be the embedding induced by the inclusion $K_{\beta_{i}} \rightarrow K\left(\beta_{1}, \ldots, \beta_{m}\right)$ onto the $i$ th factor group, and put

$$
y_{i}=\varphi_{i_{*}}\left(\omega_{\beta_{i}}\right) \in H_{2}\left(\Gamma\left(\beta_{1}, \ldots, \beta_{m}\right)\right), \quad 1 \leqslant i \leqslant m .
$$

Form the $m \times m$ strictly upper triangular matrix $A=\left(a_{i, j}\right)_{m \times m}$ by letting

$$
a_{i, j}= \begin{cases}0 & \text { if } i \geqslant j, \\ -\beta_{j} \circ \beta_{i} & \text { if } i<j .\end{cases}
$$

Lemma 2 (Bott-Samelson [BS58]). The set $\left\{y_{1}, \ldots, y_{m}\right\}$ forms a basis for $H_{2}\left(\Gamma\left(\beta_{1}, \ldots, \beta_{m}\right)\right)$. Further, let $x_{i} \in H^{2}\left(\Gamma\left(\beta_{1}, \ldots, \beta_{m}\right)\right), 1 \leqslant i \leqslant m$, be the classes Kronecker dual to $y_{1}, \ldots, y_{m}$ as $\left\langle x_{i}, y_{j}\right\rangle=\delta_{i, j}, 1 \leqslant i, j \leqslant m$, then

$$
H^{*}\left(\Gamma\left(\beta_{1}, \ldots, \beta_{m}\right)\right)=\mathbb{Z}\left[x_{1}, \ldots, x_{m}\right] / I
$$

where $I$ is the idea generated by $x_{j}^{2}-\sum_{i<j} a_{i, j} x_{i} x_{j}, 1 \leqslant j \leqslant m$.

In view of Lemma 2 one may introduce an additive map $\int_{\Gamma\left(\beta_{1}, \ldots, \beta_{m}\right)}: \mathbb{Z}\left[x_{1}, \ldots, x_{m}\right]^{(m)} \rightarrow \mathbb{Z}$ by

$$
\int_{\Gamma\left(\beta_{1}, \ldots, \beta_{m}\right)} h=\left\langle p_{\Gamma\left(\beta_{1}, \ldots, \beta_{m}\right)}(h),\left[\Gamma\left(\beta_{1}, \ldots, \beta_{m}\right)\right]\right\rangle,
$$

where $\left[\Gamma\left(\beta_{1}, \ldots, \beta_{m}\right)\right] \in H_{2 m}\left(\Gamma\left(\beta_{1}, \ldots, \beta_{m}\right)\right)=\mathbb{Z}$ is the orientation class and where

$$
p_{\Gamma\left(\beta_{1}, \ldots, \beta_{m}\right)}: \mathbb{Z}\left[x_{1}, \ldots, x_{m}\right] \rightarrow H^{*}\left(\Gamma\left(\beta_{1}, \ldots, \beta_{m}\right)\right)
$$

is the obvious quotient homomorphism in view of Lemma 2. The geometric implication of the operator $T_{A}$ in Definition 3 is seen from the next result.

Lemma 3 (Duan [Dua03a, Proposition 2]). We have

$$
\int_{\Gamma\left(\beta_{1}, \ldots, \beta_{m}\right)}=T_{A}: \mathbb{Z}\left[x_{1}, \ldots, x_{m}\right]^{(m)} \rightarrow \mathbb{Z} .
$$

In particular, $\int_{\Gamma\left(\beta_{1}, \ldots, \beta_{m}\right)} x_{1} \cdots x_{m}=1$.

It also follows from Lemma 2 that the ring $H^{*}\left(\Gamma\left(\beta_{1}, \ldots, \beta_{m}\right)\right)$ has the additive basis $\left\{x_{i_{1}} \cdots x_{i_{r}} \mid\right.$ $\left.\left[i_{1}, \ldots, i_{r}\right] \subseteq[1, \ldots, m]\right\}$. Since the dimension of each $x_{i}$ is 2 , the action of the $\mathcal{P}^{k} \in \mathcal{A}_{p}$ on these base elements is determined by the Cartan formula [SE62]. Let $m_{k, p}\left(x_{i_{1}}, \ldots, x_{i_{r}}\right)$ be the monomial symmetric function in $x_{i_{1}}, \ldots, x_{i_{r}}$ associated to the partition $\left(p^{k}, 1^{r-k}\right)$ (cf. Definition 2).

Lemma 4. We have $\mathcal{P}^{k}\left(x_{i_{1}} \cdots x_{i_{r}}\right) \equiv m_{k, p}\left(x_{i_{1}}, \ldots, x_{i_{r}}\right) \bmod p$.

Let $P$ be the centralizer of a one-parameter subgroup in $G$ and let $G / P$ be the flag manifold of left cosets of $P$ in $G$. Assume (without loss the generality) that with respect to the fixed maximal torus $T \subset G, T \subseteq P \subset G$.

Definition 4. The map

$$
\varphi_{\beta_{1}, \ldots, \beta_{m} ; P}: \Gamma\left(\beta_{1}, \ldots, \beta_{m}\right) \rightarrow G / P
$$

by $\left[g_{1}, \ldots, g_{m}\right] \rightarrow g_{1} \cdots g_{m} P$ is clearly well defined and will be called the $K$-cycle of Bott-Samelson on $G / P$ associated to the sequence $\beta_{1}, \ldots, \beta_{m} \in \Delta$ of simple roots (cf. [Dua05, $\left.\S 7.1\right]$ ).

It was first shown by Hansen [Han73] in 1972 that, when $P=T$, certain $K$-cycles provide desingularizations of Schubert varieties on $G / T$. The following more general result allows one to bring the calculation of $\mathcal{P}^{k}$-action on $H^{*}(G / P)$ (i.e. Problem B) to the computation of the action on the truncated polynomial algebra $H^{*}\left(\Gamma\left(\beta_{1}, \ldots, \beta_{m}\right)\right)$, while the latter is handled by Lemma 4 . 


\section{Formula For SteEnrod operations}

Lemma 5. Let $\left\{\sigma_{u} \in H^{2 \ell(w)}(G / P) \mid u \in \bar{W}\right\}$ be the set of Schubert classes on $G / P$ (cf. Lemma 1). The induced cohomology ring map $\varphi_{\beta_{1}, \ldots, \beta_{m} ; P}^{*}$ is given by

$$
\varphi_{\beta_{1}, \ldots, \beta_{m} ; P}^{*}\left(\sigma_{u}\right)=(-1)^{r} \sum_{\substack{J=\left[i_{1}, \ldots, i_{r}\right] \subseteq[1, \ldots, m] \\ s_{J}=u}} x_{i_{1}} \cdots x_{i_{r}},
$$

where $r=\ell(u)$.

Proof. Since $T \subseteq P \subset G$, the map $\varphi_{\beta_{1}, \ldots, \beta_{m} ; P}$ factors through $\varphi_{\beta_{1}, \ldots, \beta_{m} ; T}$ in the fashion

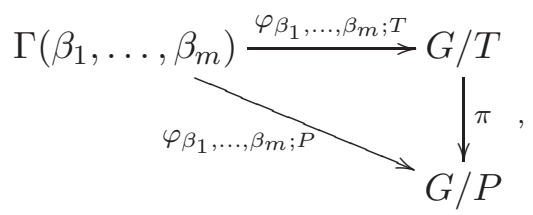

where $\pi$ is the standard fibration with fiber $P / T$. By [Dua05, Lemma 5.1] we have

(a) Lemma 5 holds for the case $P=T$.

From $[$ BGG73, $\S 5]$ we find that:

(b) the induced map $\pi^{*}: H^{*}(G / P) \rightarrow H^{*}(G / T)$ is given by

$$
\pi^{*}\left(\sigma_{w}\right)=\sigma_{w}, \quad w \in \bar{W} \subset W .
$$

Combining parts (a) and (b) verifies Lemma 5.

In the case $P=T$, a result similar to Lemma 5 in the setting of $T$-equivariant cohomology was obtained by Willems in [Wil04, Proposition 3.10].

\section{Proof of the Theorem}

For a $u \in \bar{W}$ with $\ell(u)=r$ and a $k \geqslant 1$, we assume as in (1.2) that

$$
\mathcal{P}^{k}\left(\sigma_{u}\right) \equiv \sum_{v \in \bar{W}, \ell(v)=r+p(k-1),} a_{u, v}^{k} \sigma_{v}, a_{u, v}^{k} \in \mathbb{F}_{p} .
$$

Let $w=s_{\beta_{1}} \circ \cdots \circ s_{\beta_{m}}, \beta_{i} \in \Delta$, be a reduced decomposition of a $w \in \bar{W}$ with $m=r+k(p-1)$, and let $A_{w}=\left(a_{i, j}\right)_{m \times m}$ be the associated Cartan matrix. Consider the $K$-cycle $\varphi_{\beta_{1}, \ldots, \beta_{m} ; P}$ : $\Gamma\left(\beta_{1}, \ldots, \beta_{m}\right) \rightarrow G / P$ associated to the ordered sequence $\left(\beta_{1}, \ldots, \beta_{m}\right)$ of simple roots. Applying the ring map $\varphi_{\beta_{1}, \ldots, \beta_{m} ; P}^{*}$ to Equation $(4.1)$ in $H^{*}\left(G / P ; \mathbb{F}_{p}\right)$ yields in $H^{*}\left(\Gamma\left(\beta_{1}, \ldots, \beta_{m}\right) ; \mathbb{F}_{p}\right)$ that

$$
\begin{aligned}
\varphi_{\beta_{1}, \ldots, \beta_{m} ; P}^{*} \mathcal{P}^{k}\left(\sigma_{u}\right) & \equiv \varphi_{\beta_{1}, \ldots, \beta_{m} ; P}^{*}\left(\sum_{v \in \bar{W}, \ell(v)=r+p(k-1),} a_{u, v}^{k} \sigma_{v}\right) \\
& \equiv(-1)^{m} a_{u, w}^{k} x_{1} \cdots x_{m},
\end{aligned}
$$

where the second equality follows from

$$
\varphi_{\beta_{1}, \ldots, \beta_{m} ; P}^{*}\left(\sigma_{v}\right)= \begin{cases}(-1)^{m} x_{1} \cdots x_{m} & \text { if } v=w \\ 0 & \text { if } v \neq w\end{cases}
$$


by Lemma 5 . On the other hand

$$
\begin{aligned}
& \varphi_{\beta_{1}, \ldots, \beta_{m} ; P}^{*} \mathcal{P}^{k}\left(\sigma_{u}\right) \equiv \mathcal{P}^{k}\left(\varphi_{\beta_{1}, \ldots, \beta_{m} ; P}^{*}\left(\sigma_{u}\right)\right) \\
& \equiv(-1)^{r} \mathcal{P}^{k}\left(\sum_{\substack{J=\left[i_{1}, \ldots, i_{r}\right] \subseteq[1, \ldots, m] \\
s_{J}=u}} x_{i_{1}} \cdots x_{i_{r}}\right) \quad \text { (by Lemma 5) } \\
& \equiv(-1)^{r} \sum_{\substack{J=\left[i_{1}, \ldots, i_{r}\right] \subseteq \bar{c} \\
s_{J}=\underline{u}}} m_{k, p}\left(x_{i_{1}}, \ldots, x_{i_{r}}\right) \quad \text { (by Lemma 4), }
\end{aligned}
$$

where the first equality comes from the naturality of $\mathcal{P}^{k}$ (see [SE62]). Summarizing, we get in

$$
H^{2 m}\left(\Gamma\left(\beta_{1}, \ldots, \beta_{m}\right) ; \mathbb{F}_{p}\right)=\mathbb{F}_{p}
$$

that

$$
\sum_{J=\left[i_{1}, \ldots, i_{r}\right] \subseteq[1, \ldots, m]} m_{k, p}\left(x_{i_{1}}, \ldots, x_{i_{r}}\right) \equiv(-1)^{k(p-1)} a_{u, w}^{k} x_{1} \cdots x_{m} .
$$

Evaluating both sides on the orientation class $\left[\Gamma\left(\beta_{1}, \ldots, \beta_{m}\right)\right] \bmod p$ and noting that $p$ is a prime, we get from Lemma 3 that

$$
\begin{aligned}
& a_{u, w}^{k} \equiv\left\langle\sum_{\substack{J=\left[i_{1}, \ldots, i_{r}\right] \subseteq \\
s_{J}=\underline{u}}} m_{k, p}\left(x_{i_{1}}, \ldots, x_{i_{r}}\right),\left[\Gamma\left(\beta_{1}, \ldots, \beta_{m}\right)\right]\right\rangle \\
& \equiv T_{A_{w}}\left(\sum_{\substack{J=\left[i_{1}, \ldots, i_{r}\right] \subseteq\left[ \\
s_{J}=u\right.}} m_{k, p}\left(x_{i_{1}}, \ldots, x_{i_{r}}\right)\right) .
\end{aligned}
$$

This completes the proof.

\section{Applications}

The formula (2.2) handles Problem $\mathrm{B}^{\prime}$ in its natural generality in the sense that it applies uniformly to:

(1) every flag manifold $G / P$;

(2) each Schubert class in a given $G / P$;

and, in addition, it is

(3) valid for every $k \geqslant 1$ and a prime $p \geqslant 2$.

Owing to of these features, a single program can be developed to perform computations in various $G / P$ (cf. the discussion at the end of $\S 2$ ). We list computational results from the program for some cases of $G / P$.

For the Lie groups $G$ concerned in Examples 1-4 below, we assume that a set of simple roots $\Delta=\left\{\beta_{1}, \ldots, \beta_{n}\right\}$ of $G$ is given and ordered as that in [Hum72, pp. 64-75].

For the centralizer $P$ of a one-parameter subgroup in $G$, write $\bar{W}^{r}$ for the subset of $\bar{W}=W / W^{\prime}$ consisting of the elements of length $r$ (cf. Definition 1), where $W$ (respectively $W^{\prime}$ ) is the Weyl group of $G$ (respectively $P$ ). The set $\left\{\sigma_{w} \mid w \in \bar{W}^{r}\right\}$ forms a basis for the $2 r$-dimensional cohomology $H^{2 r}(G / P)$ by Lemma 1 .

Let $\Delta_{P} \subset \Delta$ be the subset consisting of simple roots of $P$. Starting from the order on $\Delta$ as well as the subset $\Delta_{P} \subset \Delta$, a program to decompose each $w \in \bar{W}^{r}$ uniquely into a reduced product (which is the lexicographically minimum product in all reduced decompositions of $w$ ), 


\section{Formula For SteEnRod Operations}

called the minimal reduced decomposition of $w$, has been compiled in [DZ03]. If two $w, w^{\prime} \in \bar{W}^{r}$ are given by their minimal reduced decompositions

$$
w=s_{\beta_{i_{1}}} \circ \cdots \circ s_{\beta_{i_{r}}}, \quad w^{\prime}=s_{\beta_{j_{1}}} \circ \cdots \circ s_{\beta_{j_{r}}},
$$

we say $w<w^{\prime}$ if there exists a $1 \leqslant d<r$ such that $\left(i_{1}, \ldots, i_{d}\right)=\left(j_{1}, \ldots, j_{d}\right)$, but $i_{d}+1<j_{d}+1$. With respect to this order the $\bar{W}^{r}$ becomes an ordered set, hence, can be written as $\bar{W}^{r}=\left\{w_{r, i} \mid\right.$ $\left.1 \leqslant i \leqslant\left|\bar{W}^{r}\right|\right\}$.

Write $s_{i}$ instead of $s_{\beta_{i}}, \beta_{i} \in \Delta$. We list in Table 1 all $w_{r, i} \in \bar{W}$ by their minimal reduced decompositions; this followed by Table B expressing all nontrivial $\mathcal{P}^{k}\left(\sigma_{r, i}\right)$, where the notion $\sigma_{r, i}$ is used to simplify the Schubert class $\sigma_{w_{r, i}} \bmod p$.

Example 1. $G=G_{2}$ (the exceptional group of rank 2) and $H=T$ (a maximal torus).

TABLE A. Elements of $\bar{W}$ and their minimal reduced decompositions.

\begin{tabular}{llllll}
\hline$w_{r, i}$ & Decomposition & $w_{r, i}$ & Decomposition & $w_{r, i}$ & Decomposition \\
\hline$w_{1,1}$ & $s_{1}$ & $w_{3,1}$ & $s_{1} s_{2} s_{1}$ & $w_{5,1}$ & $\sigma_{1} s_{2} s_{1} s_{2} s_{1}$ \\
$w_{1,2}$ & $\sigma_{2}$ & $w_{3,2}$ & $s_{2} s_{1} s_{2}$ & $w_{5,2}$ & $s_{2} s_{1} s_{2} s_{1} s_{2}$ \\
$w_{2,1}$ & $s_{1} s_{2}$ & $w_{4,1}$ & $s_{1} s_{2} \sigma_{1} s_{2}$ & $w_{6,1}$ & $s_{1} s_{2} s_{1} \sigma_{2} s_{1} s_{2}$ \\
$w_{2,2}$ & $s_{2} s_{1}$ & $w_{4,2}$ & $s_{2} s_{1} s_{2} s_{1}$ & & \\
\hline
\end{tabular}

TABLE B. Nontrivial $P^{k}\left(\sigma_{r, i}\right)$.

\begin{tabular}{lll}
\hline$\sigma_{r, i}$ & $\mathcal{P}^{1}\left(\sigma_{r, i}\right)(p=3)$ & $\mathcal{P}^{1}\left(\sigma_{r, i}\right)(p=5)$ \\
\hline$\sigma_{1,1}$ & $2 \sigma_{3,1}$ & $2 \sigma_{5,1}$ \\
$\sigma_{1,2}$ & 0 & $3 \sigma_{5,2}$ \\
$\sigma_{2,1}$ & $2 \sigma_{4,1}$ & 0 \\
$\sigma_{2,2}$ & $\sigma_{4,2}$ & 0 \\
$\sigma_{3,1}$ & 0 & 0 \\
$\sigma_{3,2}$ & $\sigma_{5,2}$ & 0 \\
\hline
\end{tabular}

Example 2. $G=F_{4}$ (the exceptional group of rank 4) and $H=\operatorname{Spin}(7) \times S^{1}$.

TABle A. Elements of $\bar{W}$ and their minimal reduced decompositions.

\begin{tabular}{ll}
\hline$w_{r, i}$ & Decomposition \\
\hline$w_{1,1}$ & $s_{1}$ \\
$w_{2,1}$ & $s_{2} s_{1}$ \\
$w_{3,1}$ & $s_{3} s_{2} s_{1}$ \\
$w_{4,1}$ & $s_{2} s_{3} s_{2} s_{1}$ \\
$w_{4,2}$ & $s_{4} s_{3} s_{2} s_{1}$ \\
$w_{5,1}$ & $s_{1} s_{2} s_{3} s_{2} s_{1}$ \\
$w_{5,2}$ & $s_{2} s_{4} s_{3} s_{2} s_{1}$ \\
$w_{6,1}$ & $s_{1} s_{2} s_{4} s_{3} s_{2} s_{1}$ \\
$w_{6,2}$ & $s_{3} s_{2} s_{4} s_{3} s_{2} s_{1}$ \\
$w_{7,1}$ & $s_{1} s_{3} s_{2} s_{4} s_{3} s_{2} s_{1}$ \\
$w_{7,2}$ & $s_{2} s_{3} s_{2} s_{4} s_{3} s_{2} s_{1}$ \\
$w_{8,1}$ & $s_{1} s_{2} s_{3} s_{2} s_{4} s_{3} s_{2} s_{1}$ \\
\hline
\end{tabular}

\begin{tabular}{ll}
\hline$w_{r, i}$ & Decomposition \\
\hline$w_{8,2}$ & $s_{2} s_{1} s_{3} s_{2} s_{4} s_{3} s_{2} s_{1}$ \\
$w_{9,1}$ & $s_{1} s_{2} s_{1} s_{3} s_{2} s_{4} s_{3} s_{2} s_{1}$ \\
$w_{9,2}$ & $s_{3} s_{2} s_{1} s_{3} s_{2} s_{4} s_{3} s_{2} s_{1}$ \\
$w_{10,1}$ & $s_{1} s_{3} s_{2} s_{1} s_{3} s_{2} s_{4} s_{3} s_{2} s_{1}$ \\
$w_{10,2}$ & $s_{4} s_{3} s_{2} s_{1} s_{3} s_{2} s_{4} s_{3} s_{2} s_{1}$ \\
$w_{11,1}$ & $s_{1} s_{4} s_{3} s_{2} s_{1} s_{3} s_{2} s_{4} s_{3} s_{2} s_{1}$ \\
$w_{11,2}$ & $s_{2} s_{1} s_{3} s_{2} s_{1} s_{3} s_{2} s_{4} s_{3} s_{2} s_{1}$ \\
$w_{12,1}$ & $s_{2} s_{1} s_{4} s_{3} s_{2} s_{1} s_{3} s_{2} s_{4} s_{3} s_{2} s_{1}$ \\
$w_{13,1}$ & $s_{3} s_{2} s_{1} s_{4} s_{3} s_{2} s_{1} s_{3} s_{2} s_{4} s_{3} s_{2} s_{1}$ \\
$w_{14,1}$ & $s_{2} s_{3} s_{2} s_{1} s_{4} s_{3} s_{2} s_{1} s_{3} s_{2} s_{4} s_{3} s_{2} s_{1}$ \\
$w_{15,1}$ & $s_{1} s_{2} s_{3} s_{2} s_{1} s_{4} s_{3} s_{2} s_{1} s_{3} s_{2} s_{4} s_{3} s_{2} s_{1}$ \\
& \\
\hline
\end{tabular}


TABLE B. Nontrivial $P^{k}\left(\sigma_{r, i}\right)$.

\begin{tabular}{|c|c|c|c|c|c|c|}
\hline \multirow[b]{2}{*}{$\sigma_{r, i}$} & \multicolumn{2}{|c|}{$p=3$} & \multicolumn{2}{|c|}{$p=5$} & \multicolumn{2}{|c|}{$p=7$} \\
\hline & $\mathcal{P}^{1}\left(\sigma_{r, i}\right)$ & $\mathcal{P}^{2}\left(\sigma_{r, i}\right)$ & $\mathcal{P}^{1}\left(\sigma_{r, i}\right)$ & $\mathcal{P}^{2}\left(\sigma_{r, i}\right)$ & $\mathcal{P}^{1}\left(\sigma_{r, i}\right)$ & $\mathcal{P}^{2}\left(\sigma_{r, i}\right)$ \\
\hline$\sigma_{1,1}$ & $2 \sigma_{3,1}$ & 0 & $2 \sigma_{5,1}+3 \sigma_{5,2}$ & 0 & $5 \sigma_{7,1}+2 \sigma_{7,2}$ & 0 \\
\hline$\sigma_{2,1}$ & $\sigma_{4,1}+2 \sigma_{4,2}$ & $\sigma_{6,2}$ & $4 \sigma_{6,1}+2 \sigma_{6,2}$ & $3 \sigma_{10,1}+4 \sigma_{10,2}$ & $4 \sigma_{8,1}+3 \sigma_{8,2}$ & $\sigma_{14,1}$ \\
\hline$\sigma_{3,1}$ & 0 & 0 & $4 \sigma_{7,2}$ & $2 \sigma_{11,2}$ & $\sigma_{9,2}$ & $3 \sigma_{15,1}$ \\
\hline$\sigma_{4,1}$ & $\sigma_{6,2}$ & 0 & $3 \sigma_{8,1}+\sigma_{8,2}$ & $4 \sigma_{12,1}$ & $3 \sigma_{10,1}+6 \sigma_{10,2}$ & \\
\hline$\sigma_{4,2}$ & $2 \sigma_{6,2}$ & 0 & $3 \sigma_{8,1}+3 \sigma_{8,2}$ & $2 \sigma_{12,1}$ & $5 \sigma_{10,1}+3 \sigma_{10,2}$ & \\
\hline$\sigma_{5,1}$ & $\sigma_{7,1}$ & 0 & $4 \sigma_{9,1}+\sigma_{9,2}$ & $2 \sigma_{13,1}$ & $5 \sigma_{11,2}$ & \\
\hline$\sigma_{5,2}$ & $\sigma_{7,2}$ & 0 & $4 \sigma_{9,1}+\sigma_{9,2}$ & $2 \sigma_{13,1}$ & $4 \sigma_{11,1}+5 \sigma_{11,2}$ & \\
\hline$\sigma_{6,1}$ & $\sigma_{8,1}+2 \sigma_{8,2}$ & $\sigma_{10,1}+\sigma_{10,2}$ & $2 \sigma_{10,1}+2 \sigma_{10,2}$ & $4 \sigma_{14,1}$ & $3 \sigma_{12,1}$ & \\
\hline$\sigma_{6,2}$ & 0 & 0 & $4 \sigma_{10,1}$ & $2 \sigma_{14,1}$ & $\sigma_{12,1}$ & \\
\hline$\sigma_{7,1}$ & 0 & 0 & $\sigma_{11,1}+\sigma_{11,2}$ & 0 & $\sigma_{13,1}$ & \\
\hline$\sigma_{7,2}$ & 0 & 0 & $\sigma_{11,2}$ & $3 \sigma_{15,1}$ & $\sigma_{13,1}$ & \\
\hline$\sigma_{8,2}$ & $2 \sigma_{10,1}+\sigma_{10,2}$ & 0 & $3 \sigma_{12,1}$ & & $3 \sigma_{14,1}$ & \\
\hline$\sigma_{9,1}$ & $\sigma_{11,1}+2 \sigma_{11,2}$ & 0 & $\sigma_{13,1}$ & & $2 \sigma_{15,1}$ & \\
\hline$\sigma_{9,2}$ & $2 \sigma_{11,1}+2 \sigma_{11,2}$ & 0 & 0 & & $3 \sigma_{15,1}$ & \\
\hline$\sigma_{10,1}$ & $2 \sigma_{12,1}$ & $2 \sigma_{14,1}$ & $\sigma_{14,1}$ & & & \\
\hline$\sigma_{10,2}$ & $2 \sigma_{12,1}$ & $2 \sigma_{14,1}$ & $3 \sigma_{14,1}$ & & & \\
\hline$\sigma_{11,2}$ & 0 & 0 & $4 \sigma_{15,1}$ & & & \\
\hline$\sigma_{12,1}$ & $2 \sigma_{14,1}$ & & & & & \\
\hline$\sigma_{13,1}$ & $2 \sigma_{15,1}$ & & & & & \\
\hline
\end{tabular}

Example 3. $G=\mathrm{SO}(12)$ (the special orthogonal group of order 12) and $H=U(6)$. The flag manifold $G / H$ is the Grassmannian of complex structures on the 12 -dimensional real Euclidean space $\mathbb{R}^{12}$ (see [Dua02]).

TABle A. Elements of $\bar{W}$ and their minimal reduced decompositions.

\begin{tabular}{ll}
\hline$w_{r, i}$ & Decomposition \\
\hline$w_{1,1}$ & $s_{6}$ \\
$w_{2,1}$ & $s_{4} s_{6}$ \\
$w_{3,1}$ & $s_{3} s_{4} s_{6}$ \\
$w_{3,2}$ & $s_{5} s_{4} s_{6}$ \\
$w_{4,1}$ & $s_{2} s_{3} s_{4} s_{6}$ \\
$w_{4,2}$ & $s_{3} s_{5} s_{4} s_{6}$ \\
$w_{5,1}$ & $s_{1} s_{2} s_{3} s_{4} s_{6}$ \\
$w_{5,2}$ & $s_{2} s_{3} s_{5} s_{4} s_{6}$ \\
$w_{5,3}$ & $s_{4} s_{3} s_{5} s_{4} s_{6}$ \\
$w_{6,1}$ & $s_{1} s_{2} s_{3} s_{5} s_{4} s_{6}$ \\
$w_{6,2}$ & $s_{2} s_{4} s_{3} s_{5} s_{4} s_{6}$ \\
$w_{6,3}$ & $s_{6} s_{4} s_{3} s_{5} s_{4} s_{6}$ \\
$w_{7,1}$ & $s_{1} s_{2} s_{4} s_{3} s_{5} s_{4} s_{6}$ \\
$w_{7,2}$ & $s_{2} s_{6} s_{4} s_{3} s_{5} s_{4} s_{6}$ \\
$w_{7,3}$ & $s_{3} s_{2} s_{4} s_{3} s_{5} s_{4} s_{6}$ \\
$w_{8,1}$ & $s_{1} s_{2} s_{6} s_{4} s_{3} s_{5} s_{4} s_{6}$ \\
\hline
\end{tabular}

\begin{tabular}{ll}
\hline$w_{r, i}$ & Decomposition \\
\hline$w_{8,2}$ & $s_{1} s_{3} s_{2} s_{4} s_{3} s_{5} s_{4} s_{6}$ \\
$w_{8,3}$ & $s_{3} s_{2} s_{6} s_{4} s_{3} s_{5} s_{4} s_{6}$ \\
$w_{9,1}$ & $s_{1} s_{3} s_{2} s_{6} s_{4} s_{3} s_{5} s_{4} s_{6}$ \\
$w_{9,2}$ & $s_{2} s_{1} s_{3} s_{2} s_{4} s_{3} s_{5} s_{4} s_{6}$ \\
$w_{9,3}$ & $s_{4} s_{3} s_{2} s_{6} s_{4} s_{3} s_{5} s_{4} s_{6}$ \\
$w_{10,1}$ & $s_{1} s_{4} s_{3} s_{2} s_{6} s_{4} s_{3} s_{5} s_{4} s_{6}$ \\
$w_{10,2}$ & $s_{2} s_{1} s_{3} s_{2} s_{6} s_{4} s_{3} s_{5} s_{4} s_{6}$ \\
$w_{10,3}$ & $s_{5} s_{4} s_{3} s_{2} s_{6} s_{4} s_{3} s_{5} s_{4} s_{6}$ \\
$w_{11,1}$ & $s_{1} s_{5} s_{4} s_{3} s_{2} s_{6} s_{4} s_{3} s_{5} s_{4} s_{6}$ \\
$w_{11,2}$ & $s_{2} s_{1} s_{4} s_{3} s_{2} s_{6} s_{4} s_{3} s_{5} s_{4} s_{6}$ \\
$w_{12,1}$ & $s_{2} s_{1} s_{5} s_{4} s_{3} s_{2} s_{6} s_{4} s_{3} s_{5} s_{4} s_{6}$ \\
$w_{12,2}$ & $s_{3} s_{2} s_{1} s_{4} s_{3} s_{2} s_{6} s_{4} s_{3} s_{5} s_{4} s_{6}$ \\
$w_{13,1}$ & $s_{3} s_{2} s_{1} s_{5} s_{4} s_{3} s_{2} s_{6} s_{4} s_{3} s_{5} s_{4} s_{6}$ \\
$w_{14,1}$ & $s_{4} s_{3} s_{2} s_{1} s_{5} s_{4} s_{3} s_{2} s_{6} s_{4} s_{3} s_{5} s_{4} s_{6}$ \\
$w_{15,1}$ & $s_{6} s_{4} s_{3} s_{2} s_{1} s_{5} s_{4} s_{3} s_{2} s_{6} s_{4} s_{3} s_{5} s_{4} s_{6}$ \\
& \\
\hline
\end{tabular}




\section{Formula For SteEnRod operations}

TABLE $\mathrm{B}_{1}$. Nontrivial $P^{k}\left(\sigma_{r, i}\right)$ for $p=3$.

\begin{tabular}{llllll}
\hline$\sigma_{r, i}$ & $\mathcal{P}^{1}\left(\sigma_{r, i}\right)$ & $\mathcal{P}^{2}\left(\sigma_{r, i}\right)$ & $\mathcal{P}^{3}\left(\sigma_{r, i}\right)$ & $\mathcal{P}^{4}\left(\sigma_{r, i}\right)$ & $\mathcal{P}^{5}\left(\sigma_{r, i}\right)$ \\
\hline$\sigma_{1,1}$ & $\sigma_{3,1}+\sigma_{3,2}$ & 0 & 0 & 0 & 0 \\
$\sigma_{2,1}$ & $2 \sigma_{4,1}+\sigma_{4,2}$ & $\sigma_{6,1}+2 \sigma_{6,2}+2 \sigma_{6,3}$ & 0 & 0 & 0 \\
$\sigma_{3,1}$ & $\sigma_{5,2}$ & $2 \sigma_{7,1}+2 \sigma_{7,2}+2 \sigma_{7,3}$ & $\sigma_{9,1}+\sigma_{9,2}+2 \sigma_{9,3}$ & 0 & 0 \\
$\sigma_{3,2}$ & $2 \sigma_{5,2}$ & $\sigma_{7,1}+\sigma_{7,2}+\sigma_{7,3}$ & $2 s_{9,1}+\sigma_{9,2}+\sigma_{9,3}$ & 0 & 0 \\
$\sigma_{4,1}$ & $\sigma_{6,1}$ & $2 \sigma_{8,1}+2 \sigma_{8,2}$ & $2 s_{10,1}+\sigma_{10,2}$ & $2 \sigma_{12,2}$ & 0 \\
$\sigma_{4,2}$ & $\sigma_{6,2}+\sigma_{6,3}$ & $2 \sigma_{8,1}+2 \sigma_{8,2}$ & $2 \sigma_{10,1}+2 \sigma_{10,2}$ & $\sigma_{12,1}$ & 0 \\
$\sigma_{5,2}$ & $\sigma_{7,1}+\sigma_{7,2}+\sigma_{7,3}$ & 0 & $\sigma_{11,2}$ & $s_{13,1}$ & $2 \sigma_{15,1}$ \\
$\sigma_{5,3}$ & $\sigma_{7,2}+2 \sigma_{7,3}$ & $\sigma_{9,2}$ & $\sigma_{11,2}$ & $s_{13,1}$ & $2 \sigma_{15,1}$ \\
$\sigma_{6,1}$ & $\sigma_{8,1}+\sigma_{8,2}$ & 0 & 0 & 0 & \\
$\sigma_{6,2}$ & $\sigma_{8,1}+\sigma_{8,2}+\sigma_{8,3}$ & $2 \sigma_{10,1}+2 s_{10,2}+2 \sigma_{10,3}$ & $\sigma_{12,1}+\sigma_{12,2}$ & 0 & \\
$\sigma_{6,3}$ & $2 \sigma_{8,3}$ & $\sigma_{10,1}+\sigma_{10,2}+\sigma_{10,3}$ & $s_{12,1}+\sigma_{12,2}$ & 0 & \\
$\sigma_{7,1}$ & $\sigma_{9,1}+2 \sigma_{9,2}$ & $2 \sigma_{11,1}+2 \sigma_{11,2}$ & 0 & 0 & \\
$\sigma_{7,2}$ & $\sigma_{9,1}$ & $2 \sigma_{11,1}+2 \sigma_{11,2}$ & $2 s_{13,1}$ & $2 \sigma_{15,1}$ & \\
$\sigma_{7,3}$ & $\sigma_{9,1}+\sigma_{9,2}$ & $2 \sigma_{11,1}+2 \sigma_{11,2}$ & 0 & & \\
$\sigma_{8,1}$ & $2 \sigma_{10,2}$ & $\sigma_{12,1}+\sigma_{12,2}$ & $\sigma_{14,1}$ & \\
$\sigma_{8,2}$ & $\sigma_{10,2}$ & $2 \sigma_{12,1}+2 \sigma_{12,2}$ & $2 s_{14,1}$ & \\
$\sigma_{8,3}$ & $\sigma_{10,1}+\sigma_{10,2}+\sigma_{10,3}$ & 0 & $\sigma_{14,1}$ & \\
$\sigma_{9,1}$ & $\sigma_{11,1}+\sigma_{11,2}$ & 0 & 0 & \\
$\sigma_{9,3}$ & $\sigma_{11,1}+\sigma_{11,2}$ & 0 & $\sigma_{15,1}$ & \\
$\sigma_{10,1}$ & $\sigma_{12,1}+2 \sigma_{12,2}$ & 0 & & \\
$\sigma_{10,2}$ & $\sigma_{12,1}+\sigma_{12,2}$ & 0 & & \\
$\sigma_{10,3}$ & $\sigma_{12,1}$ & 0 & & \\
$\sigma_{11,1}$ & $2 \sigma_{13,1}$ & $\sigma_{15,1}$ & & \\
$\sigma_{11,2}$ & $\sigma_{13,1}$ & $2 \sigma_{15,1}$ & & \\
$\sigma_{13,1}$ & $\sigma_{15,1}$ & & & \\
\hline
\end{tabular}

TABLE $\mathrm{B}_{2}$. Nontrivial $P^{k}\left(\sigma_{r, i}\right)$ for $p=5$.

\begin{tabular}{llll}
\hline$\sigma_{r, i}$ & $\mathcal{P}^{1}\left(\sigma_{r, i}\right)$ & $\mathcal{P}^{2}\left(\sigma_{r, i}\right)$ & $\mathcal{P}^{3}\left(\sigma_{r, i}\right)$ \\
\hline$\sigma_{1,1}$ & $\sigma_{5,1}+3 \sigma_{5,2}+2 \sigma_{5,3}$ & 0 & 0 \\
$\sigma_{2,1}$ & $3 \sigma_{6,1}+4 \sigma_{6,3}$ & 0 & 0 \\
$\sigma_{3,1}$ & $4 \sigma_{7,2}+3 \sigma_{7,3}$ & $2 \sigma_{11,1}$ & $4 s_{15,1}$ \\
$\sigma_{3,2}$ & $2 \sigma_{7,1}+2 \sigma_{7,2}+2 \sigma_{7,3}$ & $\sigma_{11,1}$ & $2 \sigma_{15,1}$ \\
$\sigma_{4,1}$ & $4 \sigma_{8,1}+3 \sigma_{8,2}$ & $\sigma_{12,2}$ & \\
$\sigma_{4,2}$ & $4 \sigma_{8,2}+4 \sigma_{8,3}$ & $3 \sigma_{12,1}+2 s_{12,2}$ & \\
$\sigma_{5,2}$ & $2 \sigma_{9,1}+2 \sigma_{9,2}+2 \sigma_{9,3}$ & $2 s_{13,1}$ & \\
$\sigma_{5,3}$ & $2 \sigma_{9,1}+2 \sigma_{9,2}+2 \sigma_{9,3}$ & $2 s_{13,1}$ & \\
$\sigma_{6,1}$ & $2 \sigma_{10,1}+3 \sigma_{10,2}$ & 0 & \\
$\sigma_{6,2}$ & $2 \sigma_{10,1}+2 \sigma_{10,2}+4 \sigma_{10,3}$ & $4 s_{14,1}$ & \\
$\sigma_{6,3}$ & $3 \sigma_{10,1}+2 \sigma_{10,2}+\sigma_{10,3}$ & 0 & \\
$\sigma_{7,1}$ & $4 \sigma_{11,1}$ & $2 \sigma_{15,1}$ & \\
$\sigma_{7,2}$ & $3 \sigma_{11,1}$ & $4 \sigma_{15,1}$ & \\
$\sigma_{7,3}$ & $4 \sigma_{11,1}$ & $2 \sigma_{15,1}$ & \\
$\sigma_{8,1}$ & $2 \sigma_{12,1}+2 \sigma_{12,2}$ & & \\
$\sigma_{8,2}$ & $4 \sigma_{12,1}+3 \sigma_{12,2}$ & & \\
$\sigma_{8,3}$ & $3 \sigma_{12,2}$ & & \\
$\sigma_{9,1}$ & $4 \sigma_{13,1}$ & & \\
$\sigma_{9,3}$ & $3 \sigma_{13,1}$ & & \\
$\sigma_{10,1}$ & $2 \sigma_{14,1}$ & & \\
$\sigma_{10,2}$ & $2 \sigma_{14,1}$ & & \\
$\sigma_{11,1}$ & $\sigma_{15,1}$ & \\
$\sigma_{11,2}$ & $4 \sigma_{15,1}$ & & \\
\hline
\end{tabular}




\section{H. DUAN AND X. ZHAO}

Example 4. $G=\mathrm{U}(7)$ (the unitary group of order 7) and $H=\mathrm{U}(3) \times \mathrm{U}(4)$. The flag manifold $G / H$ is the Grassmannian of 3-planes through the origin in $\mathbb{C}^{7}$.

TABle A. Elements of $\bar{W}$ and their minimal reduced decompositions.

\begin{tabular}{|c|c|c|c|c|c|}
\hline$w_{r, i}$ & Decomposition & $w_{r, i}$ & Decomposition & $w_{r, i}$ & Decomposition \\
\hline$w_{1,1}$ & $s_{3}$ & $w_{5,3}$ & $s_{2} s_{6} s_{5} s_{4} s_{3}$ & $w_{8,1}$ & $s_{1} s_{4} s_{3} s_{2} s_{6} s_{5} s_{4} s_{3}$ \\
\hline$w_{2,1}$ & $s_{2} s_{3}$ & $w_{5,4}$ & $s_{3} s_{2} s_{5} s_{4} s_{3}$ & $w_{8,2}$ & $s_{2} s_{1} s_{3} s_{2} s_{6} s_{5} s_{4} s_{3}$ \\
\hline$w_{2,2}$ & $s_{4} s_{3}$ & $w_{6,1}$ & $s_{1} s_{2} s_{6} s_{5} s_{4} s_{3}$ & $w_{8,3}$ & $s_{2} s_{1} s_{4} s_{3} s_{2} s_{5} s_{4} s_{3}$ \\
\hline$w_{3,1}$ & $s_{1} s_{2} s_{3}$ & $w_{6,2}$ & $s_{1} s_{3} s_{2} s_{5} s_{4} s_{3}$ & $w_{8,4}$ & $s_{5} s_{4} s_{3} s_{2} s_{6} s_{5} s_{4} s_{3}$ \\
\hline$w_{3,2}$ & $s_{2} s_{4} s_{3}$ & $w_{6,3}$ & $s_{2} s_{1} s_{3} s_{2} s_{4} s_{3}$ & $w_{9,1}$ & $s_{1} s_{5} s_{4} s_{3} s_{2} s_{6} s_{5} s_{4} s_{3}$ \\
\hline$w_{3,3}$ & $s_{5} s_{4} s_{3}$ & $w_{6,4}$ & $s_{3} s_{2} s_{6} s_{5} s_{4} s_{3}$ & $w_{9,2}$ & $s_{2} s_{1} s_{4} s_{3} s_{2} s_{6} s_{5} s_{4} s_{3}$ \\
\hline$w_{4,1}$ & $s_{1} s_{2} s_{4} s_{3}$ & $w_{6,5}$ & $s_{4} s_{3} s_{2} s_{5} s_{4} s_{3}$ & $w_{9,3}$ & $s_{3} s_{2} s_{1} s_{4} s_{3} s_{2} s_{5} s_{4} s_{3}$ \\
\hline$w_{4,2}$ & $s_{2} s_{5} s_{4} s_{3}$ & $w_{7,1}$ & $s_{1} s_{3} s_{2} s_{6} s_{5} s_{4} s_{3}$ & $w_{10,1}$ & $s_{2} s_{1} s_{5} s_{4} s_{3} s_{2} s_{6} s_{5} s_{4} s_{3}$ \\
\hline$w_{4,3}$ & $s_{3} s_{2} s_{4} s_{3}$ & $w_{7,2}$ & $s_{1} s_{4} s_{3} s_{2} s_{5} s_{4} s_{3}$ & $w_{10,2}$ & $s_{3} s_{2} s_{1} s_{4} s_{3} s_{2} s_{6} s_{5} s_{4} s_{3}$ \\
\hline$w_{4,4}$ & $s_{6} s_{5} s_{4} s_{3}$ & $w_{7,3}$ & $s_{2} s_{1} s_{3} s_{2} s_{5} s_{4} s_{3}$ & $w_{11,1}$ & $s_{3} s_{2} s_{1} s_{5} s_{4} s_{3} s_{2} s_{6} s_{5} s_{4} s_{3}$ \\
\hline$w_{5,1}$ & $s_{1} s_{2} s_{5} s_{4} s_{3}$ & $w_{7,4}$ & $s_{4} s_{3} s_{2} s_{6} s_{5} s_{4} s_{3}$ & $w_{12,1}$ & $s_{4} s_{3} s_{2} s_{1} s_{5} s_{4} s_{3} s_{2} s_{6} s_{5} s_{4} s_{3}$ \\
\hline$w_{5,2}$ & $s_{1} s_{3} s_{2} s_{4} s_{3}$ & & & & \\
\hline
\end{tabular}

TABle $\mathrm{B}_{1}$. Nontrivial $P^{k}\left(\sigma_{r, i}\right)$ for $p=3$.

\begin{tabular}{|c|c|c|c|c|}
\hline$\sigma_{r, i}$ & $\mathcal{P}^{1}\left(\sigma_{r, i}\right)$ & $\mathcal{P}^{2}\left(\sigma_{r, i}\right)$ & $\mathcal{P}^{3}\left(\sigma_{r, i}\right)$ & $\mathcal{P}^{4}\left(\sigma_{r, i}\right)$ \\
\hline$\sigma_{1,1}$ & $\sigma_{3,1}+2 \sigma_{3,2}+\sigma_{3,3}$ & 0 & 0 & 0 \\
\hline$\sigma_{2,1}$ & $2 \sigma_{4,1}+\sigma_{4,2}+2 \sigma_{4,3}$ & $2 \sigma_{6,2}+\sigma_{6,3}+\sigma_{6,5}$ & 0 & 0 \\
\hline$\sigma_{2,2}$ & $\sigma_{4,1}+2 \sigma_{4,2}+2 \sigma_{4,3}+2 \sigma_{4,4}$ & $s_{6,1}+2 \sigma_{6,2}+\sigma_{6,3}+\sigma_{6,5}$ & 0 & 0 \\
\hline$\sigma_{3,1}$ & $\sigma_{5,1}+2 \sigma_{5,2}$ & $\sigma_{7,2}+2 \sigma_{7,3}$ & $s_{9,3}$ & 0 \\
\hline$\sigma_{3,2}$ & $2 \sigma_{5,1}+2 \sigma_{5,2}+2 \sigma_{5,3}+2 \sigma_{5,4}$ & $2 \sigma_{7,1}+2 \sigma_{7,4}$ & $\sigma_{9,1}+2 \sigma_{9,2}+2 \sigma_{9,3}$ & 0 \\
\hline$\sigma_{3,3}$ & $\sigma_{5,1}+2 \sigma_{5,3}+2 \sigma_{5,4}$ & $2 \sigma_{7,1}+2 \sigma_{7,2}+\sigma_{7,3}+2 \sigma_{7,4}$ & $\sigma_{9,1}+2 \sigma_{9,2}+s_{9,3}$ & 0 \\
\hline$\sigma_{4,1}$ & $2 \sigma_{6,1}+2 \sigma_{6,2}$ & $\sigma_{8,1}+2 s_{8,2}+2 \sigma_{8,3}$ & $\sigma_{10,1}+\sigma_{10,2}$ & $\sigma_{12,1}$ \\
\hline$\sigma_{4,2}$ & $2 \sigma_{6,1}+2 \sigma_{6,2}+2 \sigma_{6,4}$ & $\sigma_{8,1}+2 \sigma_{8,3}+\sigma_{8,4}$ & $2 \sigma_{10,2}$ & $2 \sigma_{12,1}$ \\
\hline$\sigma_{4,3}$ & $2 \sigma_{6,2}+\sigma_{6,3}+2 \sigma_{6,4}+\sigma_{6,5}$ & $2 \sigma_{8,1}+\sigma_{8,2}+\sigma_{8,4}$ & $2 \sigma_{10,1}+\sigma_{10,2}$ & $s_{12,1}$ \\
\hline$\sigma_{4,4}$ & $\sigma_{6,1}+2 \sigma_{6,4}$ & $2 \sigma_{8,1}+\sigma_{8,2}+s_{8,4}$ & $2 \sigma_{10,1}+\sigma_{10,2}$ & $\sigma_{12,1}$ \\
\hline$\sigma_{5,1}$ & $2 \sigma_{7,1}$ & $\sigma_{9,1}+2 \sigma_{9,2}$ & 0 & \\
\hline$\sigma_{5,2}$ & $2 \sigma_{7,1}+\sigma_{7,2}+2 \sigma_{7,3}$ & $\sigma_{9,1}+2 \sigma_{9,2}$ & 0 & \\
\hline$\sigma_{5,3}$ & $2 \sigma_{7,1}$ & $\sigma_{9,1}+2 \sigma_{9,2}$ & 0 & \\
\hline$\sigma_{5,4}$ & $2 \sigma_{7,1}+2 \sigma_{7,2}+\sigma_{7,3}+2 \sigma_{7,4}$ & 0 & 0 & \\
\hline$\sigma_{6,2}$ & $2 \sigma_{8,1}+2 \sigma_{8,2}+2 \sigma_{8,3}$ & 0 & $2 s_{12,1}$ & \\
\hline$\sigma_{6,3}$ & $2 \sigma_{8,2}+\sigma_{8,3}$ & $\sigma_{10,1}$ & 0 & \\
\hline$\sigma_{6,4}$ & $2 \sigma_{8,1}+\sigma_{8,2}+\sigma_{8,4}$ & 0 & $2 s_{12,1}$ & \\
\hline$\sigma_{6,5}$ & $2 \sigma_{8,1}+\sigma_{8,3}$ & $2 \sigma_{10,1}$ & $2 s_{12,1}$ & \\
\hline$\sigma_{7,1}$ & $\sigma_{9,1}+2 \sigma_{9,2}$ & 0 & & \\
\hline$\sigma_{7,2}$ & $2 \sigma_{9,2}+2 \sigma_{9,3}$ & $2 \sigma_{11,1}$ & & \\
\hline$\sigma_{7,3}$ & $2 \sigma_{9,2}+2 \sigma_{9,3}$ & $2 \sigma_{11,1}$ & & \\
\hline$\sigma_{7,4}$ & $2 \sigma_{9,1}+\sigma_{9,2}$ & 0 & & \\
\hline$\sigma_{8,1}$ & $2 \sigma_{10,1}+2 \sigma_{10,2}$ & $\sigma_{12,1}$ & & \\
\hline$\sigma_{8,2}$ & $\sigma_{10,1}+2 \sigma_{10,2}$ & $\sigma_{12,1}$ & & \\
\hline$\sigma_{8,3}$ & $2 \sigma_{10,2}$ & $\sigma_{12,1}$ & & \\
\hline$\sigma_{8,4}$ & $\sigma_{10,1}$ & & & \\
\hline$\sigma_{9,1}$ & $2 \sigma_{11,1}$ & & & \\
\hline$\sigma_{9,2}$ & $2 \sigma_{11,1}$ & & & \\
\hline$\sigma_{10,2}$ & $\sigma_{12,1}$ & & & \\
\hline
\end{tabular}




\section{Formula For SteEnRod Operations}

TABLE $\mathrm{B}_{2}$. Nontrivial $P^{k}\left(\sigma_{r, i}\right)$ for $p=5$.

\begin{tabular}{lll}
\hline$\sigma_{r, i}$ & $\mathcal{P}^{1}\left(\sigma_{r, i}\right)$ & $\mathcal{P}^{2}\left(\sigma_{r, i}\right)$ \\
\hline$\sigma_{1,1}$ & $\sigma_{5,1}+4 \sigma_{5,3}$ & 0 \\
$\sigma_{2,1}$ & $4 \sigma_{6,1}+\sigma_{6,2}+4 \sigma_{6,4}$ & $\sigma_{10,1}$ \\
$\sigma_{2,2}$ & $\sigma_{6,1}+\sigma_{6,2}+4 \sigma_{6,4}$ & $\sigma_{10,1}$ \\
$\sigma_{3,1}$ & $4 \sigma_{7,1}+\sigma_{7,3}$ & $\sigma_{11,1}$ \\
$\sigma_{3,2}$ & $\sigma_{7,2}+\sigma_{7,3}+4 \sigma_{7,4}$ & $2 \sigma_{11,1}$ \\
$\sigma_{3,3}$ & $\sigma_{7,1}+\sigma_{7,2}+4 \sigma_{7,4}$ & $\sigma_{11,1}$ \\
$\sigma_{4,1}$ & $4 \sigma_{8,1}+\sigma_{8,2}+\sigma_{8,3}$ & $\sigma_{12,1}$ \\
$\sigma_{4,2}$ & $\sigma_{8,2}+\sigma_{8,3}+4 \sigma_{8,4}$ & $2 \sigma_{12,1}$ \\
$\sigma_{4,3}$ & $\sigma_{8,1}+4 \sigma_{8,2}+\sigma_{8,3}$ & $\sigma_{12,1}$ \\
$\sigma_{4,4}$ & $\sigma_{8,1}+4 \sigma_{8,4}$ & $\sigma_{12,1}$ \\
$\sigma_{5,1}$ & $4 \sigma_{9,1}+\sigma_{9,2}$ & \\
$\sigma_{5,2}$ & $\sigma_{9,2}+\sigma_{9,3}$ & \\
$\sigma_{5,3}$ & $4 \sigma_{9,1}+\sigma_{9,2}$ & \\
$\sigma_{5,4}$ & $\sigma_{9,1}+\sigma_{9,3}$ & \\
$\sigma_{6,2}$ & $\sigma_{10,1}+\sigma_{10,2}$ & \\
$\sigma_{6,3}$ & $2 \sigma_{10,2}$ & \\
$\sigma_{6,4}$ & $4 \sigma_{10,1}+\sigma_{10,2}$ & \\
$\sigma_{6,5}$ & $\sigma_{10,1}+4 \sigma_{10,2}$ & \\
$\sigma_{7,2}$ & $\sigma_{11,1}$ & \\
$\sigma_{7,3}$ & $2 \sigma_{11,1}$ & \\
$\sigma_{7,4}$ & $4 \sigma_{11,1}$ & \\
$\sigma_{8,3}$ & $2 \sigma_{12,1}$ & \\
$\sigma_{8,4}$ & $3 \sigma_{12,1}$ & \\
\hline
\end{tabular}

\section{ACKNOWLEDGEMENTS}

The authors are very grateful to their referee and to K. Y. Lam for many improvements on the earlier version of the paper.

\section{REFERENCES}

BGG73 I. N. Bernstein, I. M. Gel'fand and S. I. Gel'fand, Schubert cells and cohomology of the spaces $G / P$, Russian Math. Surveys 28 (1973), 1-26.

BH58 A. Borel and F. Hirzebruch, Characteristic classes and homogeneous spaces. I, Amer. J. Math. 80 (1958), 458-538.

BS51 A. Borel and J. P. Serre, Détermination des p-puissances réduites de Steenrod dans la cohomologie des groupes classiques. Applications, C. R. Acad. Sci. Paris Sér. I Math. 233 (1951), 680-682.

BS53 A. Borel and J. P. Serre, Groupes de Lie et puissances réduites de Steenrod, Amer. J. Math. 75 (1953), 409-448.

BS55 R. Bott and H. Samelson, The cohomology ring of $G / T$, Proc. Natl. Acad. Sci. USA 41 (1955), 490-493.

BS58 R. Bott and H. Samelson, Application of the theory of Morse to symmetric spaces, Amer. J. Math. 80 (1958), 964-1029.

Che94 C. Chevalley, Sur les décompositions cellulaires des espaces G/B, in Algebraic groups and their generalizations: classical methods, ed. W. Haboush, Proceedings of Symposia in Pure Mathematics, vol. 56 (part 1) (American Mathematical Society, Providence, RI, 1994), 1-26.

Die89 J. Dieudonné, A history of algebraic and differential topology, 1900-1960 (Birkhäuser, Basel, 1989). 


\section{Formula For SteEnRod OpERATIONS}

Dua02 H. Duan, Self-maps of the Grassmannian of complex structures, Compositio Math. 132 (2002), 159-175.

Dua03a H. Duan, The degree of a Schubert variety, Adv. Math. 180 (2003), 112-133.

Dua03b H. Duan, On the inverse Kostka matrix, J. Combin. Theory Ser. A 103 (2003), 363-376.

Dua05 H. Duan, Multiplicative rule of Schubert classes, Invent. Math. 159 (2005), 407-436.

DZ03 H. Duan and X. Zhao, Algorithm for multiplying Schubert classes, Internat. J. Algebra Comput., to appear.

DZZ04 H. Duan, X. Zhao and X. Zhao, The Cartan matrix and enumerative calculus, J. Symbolic Comput. 38 (2004), 1119-1144.

Ehr34 C. Ehresmann, Sur la topologie de certains espaces homogenes, Ann. of Math. (2) 35 (1934), 396-443.

GH78 P. Griffith and J. Harris, Principles of algebraic geometry (Wiley, New York, 1978).

Han73 H. C. Hansen, On cycles in flag manifolds, Math. Scand. 33 (1973), 269-274.

Hum72 J. E. Humphreys, Introduction to Lie algebras and representation theory, Graduate Texts in Mathematics, vol. 9 (Springer, New York, 1972).

Kle76 S. Kleiman, Problem 15: rigorous foundation of Schubert's enumerative calculus, Proceedings of Symposia in Pure Mathematics, vol. 28 (American Mathematical Society, Providence, RI, 1976), $445-482$.

Lan83 T. Lance, Steenrod and Dyer-Lashof operations on BU, Trans. Amer. Math. Soc. 276 (1983), 497-510.

Len98 C. Lenart, The combinatorics of Steenrod operations on the cohomology of Grassmannians, Adv. Math. 136 (1998), 251-283.

Mac95 I. G. Macdonald, Symmetric functions and Hall polynomials, Oxford Mathematical Monographs, second edition (Oxford University Press, Oxford, 1995).

Pet75 F. Peterson, A mod-p Wu formula, Bol. Soc. Mat. Mexicana (3) 20 (1975), 56-58.

Sha77 B. Shay, mod-p Wu formulas for the Steenrod algebra and the Dyer-Lashof algebra, Proc. Amer. Math. Soc. 63 (1977), 339-347.

Sot02 F. Sottile, Schubert cycles, in Encyclopaedia of Mathematics, Supplement III (Kluwer Academic, Dordrecht, 2002), 344.

Ste72 N. E. Steenrod, Cohomology operations, and obstructions to extending continuous functions, Adv. Math. 8 (1972), 371-416.

SE62 N. E. Steenrod and D. B. A. Epstein, Cohomology operations, Annals of Mathematics Studies, vol. 50 (Princeton University Press, Princeton, NJ, 1962).

SW51 N. E. Steenrod and J. H. C. Whitehead, Vector fields on the n-sphere, Proc. Natl. Acad. Sci. USA 37 (1951), 58-63.

Sug79 T. Sugawara, Wu formulas for the mod-3 reduced power operations, Mem. Fac. Sci. Kyushu Univ. Ser. A 33 (1979), 297-309.

Wil04 M. Willems, Cohomologie et K-théorie équivariantes des variétés de Bott-Samelson et des variétés de drapeaux, Bull. Soc. Math. France 132 (2004), 569-589.

Woo98 R. Wood, Problems in the Steenrod algebra, Bull. London Math. Soc. 30 (1998), 449-517.

Wu50 T. Wu, Les i-carrés dans une variété grassmannienne, C. R. Acad. Sci. Paris Sér. I Math. 230 (1950), 918-920.

Haibao Duan dhb@math.ac.cn

Institute of Mathematics, Chinese Academy of Sciences, Beijing 100080, People's Republic of China

Xuezhi Zhao zhaoxve@mail.cnu.edu.cn

Department of Mathematics, Capital Normal University, Beijing 100037, People's Republic of China 